\title{
O uso da pressão de pulso como preditor independente de mortalidade na insuficiência cardíaca
}

\author{
The use of pulse pressure as na independente predictor of mortality in heart failure \\ El uso de la presión del pulso como um predictor independiente de mortalidade em la \\ insuficiencia cardíaca
}

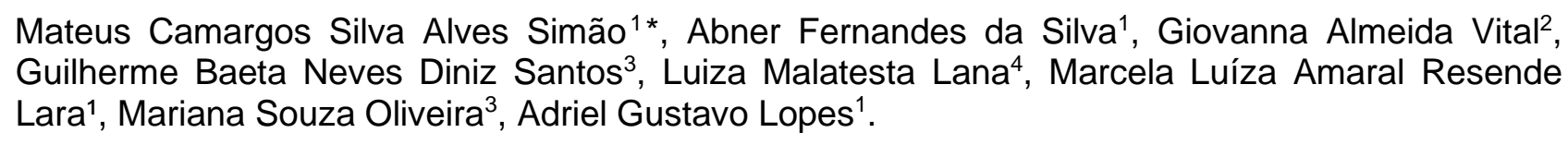

RESUMO

Objetivo: Avaliar o uso da pressão de pulso (PP) como preditor independente de mortalidade na insuficiência cardíaca (IC). Métodos: Foi realizado uma revisão integrativa, a partir de publicações indexadas nas bases de dados Medline, PubMed, Lilacs e Scielo com resultados em métodos quantitativos e qualitativos no período de 2004 a 2020, nos idiomas inglês, português e espanhol. Ademais, segundo os critérios de inclusão, foi feita uma seleção de 6 artigos selecionados para a análise. Resultados: Observou-se que na IC, a pressão de pulso baixa foi interligada a maior taxa de mortalidade em pacientes com fração de ejeção (FEVE) reduzida. Já valores altos de PP foram associados a maior taxa de óbito em pacientes com FEVE preservada. Considerações finais: Concluiu-se que a PP é um marcador prognóstico em potencial para mortalidade na insuficiência cardíaca, e pode ser implementada na prática médica de forma simples. No entanto são necessários mais estudos acerca desse assunto.

Palavras-chave: Pulso arterial, Doenças cardiovasculares, Insuficiência cardíaca, Mortalidade.

\section{ABSTRACT}

Objective: To assess the use of pulse pressure (PP) as na independente predictor of mortality in heart failure $(\mathrm{HF})$. Methods: This is an integrative review, with search performed from publications indexed in the following databases: Medline, PubMed, Lilacs and Scielo with results in quantitative and qualitative methods published between 2004 and 2020, in the languages english, portuguese and spanish. Furthermore, according to the inclusion criteria, 6 articles was picked to analysis. Results: It was observed that in HF, low PP was linked to a higher mortality rate in patients with reduced LVEF. Likewise, high PP values were associated with a higher death rate in patients with preserved LVEF. Final considerations: In conclusion, PP has shown great capability as a predictor of mortality in HF and can be implemented in medical practice in a simple way. Although, more studies are needed on this subject.

Keywords: Arterial pulse, Cardiovascular diseases, Heart failure, Mortality.

RESUMEN

Objetivo: Evaluar el uso de la presión de pulso (PP) como um predictor independiente de mortalidade em la insuficiencia cardíaca (IC). Métodos: Ha sido realizada una revisíon integradora, a partir de publicaciones indexadas em las bases de datos Medline, PubMed Lilacs y Scielo con resultados en métodos cantitativos y calitativos em el período de 2004 hasta 2020, em inglés, portugués y español. Además, según los criterios de inclusión, ha sido hecha una selección de 6 artículos para el análisis. Resultados: Se observó que em la IC, la PP baja se relacionócon una mayor tasa de mortalidade en pacientes con FEVI reducida. Los valores altos de PP se asociaron con una mayor tasa de mortalidade en pacientes con FEVI preservada. Consideraciones

\footnotetext{
${ }^{1}$ Pontifícia Universidade Católica de Minas Gerais (PUC-MG), Betim - MG.

*E-mail: camargosmateus@gmail.com

2Universidade Federal de São João del-Rei (UFSJ), São João del-Rei - MG.

${ }^{3}$ Faculdade de Minas (FAMINAS), Belo Horizonte - MG.

${ }^{4}$ Universidade Presidente Antônio Carlos (UNIPAC), Juiz de Fora - MG.
} 
finales: Se concluyó que la PP es un potencial predictor de mortalidade em la IC y puede ser implementado em la práctica médica de una manera simple. Sin embargo, se necesitan más estudios sobre este tema.

Palabras clave: Pulso arterial, Enfermedades cardiovasculares, Insuficiencia cardíaca, Mortalidad.

\section{INTRODUÇÃO}

A pressão de pulso (PP) equivale a diferença entre a pressão arterial sistólica (PAS) e a pressão arterial diastólica (PAD). Essa medida é facilmente mensurada na admissão hospitalar. Além disso, a PP tem sido considerada como preditor prognóstico para eventos cardiovasculares, quando comparados aos valores de PAS e PAD separadamente (RODRIGUES A, et al., 2015). Vários dados têm demonstrado que a PP pode ser um fator prognóstico mais relevante quando comparado às PAS, PAD e pressão arterial média para avaliar pacientes com insuficiência cardíaca (IC) (ALVIM R, et al., 2017; MONTERA MW, et al., 2009).

A partir dessa variável é possível avaliar o enrijecimento da aorta, resultado de uma elevação da PAS e diminuição da PAD. Esse fenômeno é associado ao aumento da pós-carga e hipertrofia no ventrículo esquerdo, além da diminuição da perfusão coronariana, levando então a um aumento da PP. Ademais, a pressão de pulso representa o componente pulsátil do débito cardíaco (DC), definida pela fração de ejeção em conjunto com as propriedades viscoelásticas de grandes artérias. Dessa forma, uma baixa pressão de pulso é correlacionada ao baixo débito cardíaco, à níveis aumentados de peptídeos natriuréticos (marcador de tensão na IC) e à maior taxa de mortalidade (ALVIM, et al., 2017; MONTERA, et al., 2009).

Visto a relação entre a PP e doenças cardiovasculares (DCV), é de extrema relevância ressaltar que essas são as principais causas de morte no mundo. De acordo com a Organização Mundial de Saúde, foram registrados 17,9 milhões de mortes por doenças cardiovasculares em 2016, representando $31 \%$ da mortalidade mundial. Já no Brasil, a Sociedade Brasileira de Cardiologia apontou 383.961 óbitos por DCV em 2017 e registrou 117.441 mortes até o mês de abril de 2020 (OMS, 2017; SBC, 2020).

Dentre as DCV, a IC, de acordo com a World Heart Federation, é responsável por 31.7 milhões de mortes por ano, e em âmbito nacional, o DATA-SUS registra 26.694 óbitos e cerca de 238.890 internações pela mesma condição no ano de 2012, o que representa $21 \%$ das internações por doenças do aparelho circulatório (WHF, 2019; SBC, 2015). Observada a significância da IC, torna-se necessário o estudo de preditores que possam auxiliar na avaliação desta patologia. A utilização sistemática da PP como marcador prognóstico pode ser empregada facilmente e sem custos adicionais. Sendo assim, o objetivo deste artigo é avaliar se a PP pode ser utilizada como preditor suficiente e independente da IC.

\section{METÓDOS}

Trata-se de um estudo de abordagem quantitativo, para avaliar o uso da pressão de pulso como preditor independente de mortalidade na insuficiência cardíaca. Para isso, adotou-se a revisão integrativa como desenho de estudo. Foi elaborado uma pergunta PICO e, a partir dela, feito um levantamento bibliográfico seguindo critérios bem definidos para a coleta de dados, além de uma análise e apresentação dos resultados.

Nesse objetivo, a busca foi dividida em 6 etapas: 1) elaboração de pergunta norteadora, 2) amostragem na literatura, 3) coleta de dados, 4) análise crítica dos estudos incluídos, 5) discussão dos resultados 6) apresentação da revisão integrativa. Para o levantamento dos artigos, realizou-se uma busca ativa nas seguintes bases de dados: LILACS, Medline, SciELO e PubMed, no período de 2004 a 2020.

Os critérios de inclusão definidos para a seleção dos artigos foram: artigos publicados em português, inglês e espanhol; artigos na íntegra que retratassem a temática referente à revisão integrativa, todos os desenhos de estudo (relato de experiência, original, revisão de literatura, coortes prospectivas), estudos publicados e indexados nos referidos bancos de dados nos últimos dezesseis anos, aqueles que contivessem em seus títulos e/ou resumos os seguintes descritores: "pressão de pulso", "doenças cardiovasculares", "insuficiência cardíaca" e "mortalidade". O critério de exclusão adotado foi: estudos que não atendessem os critérios de inclusão (Figura 1). 
Figura 1 - Fluxo das informações com as diferentes fases da revisão integrativa.

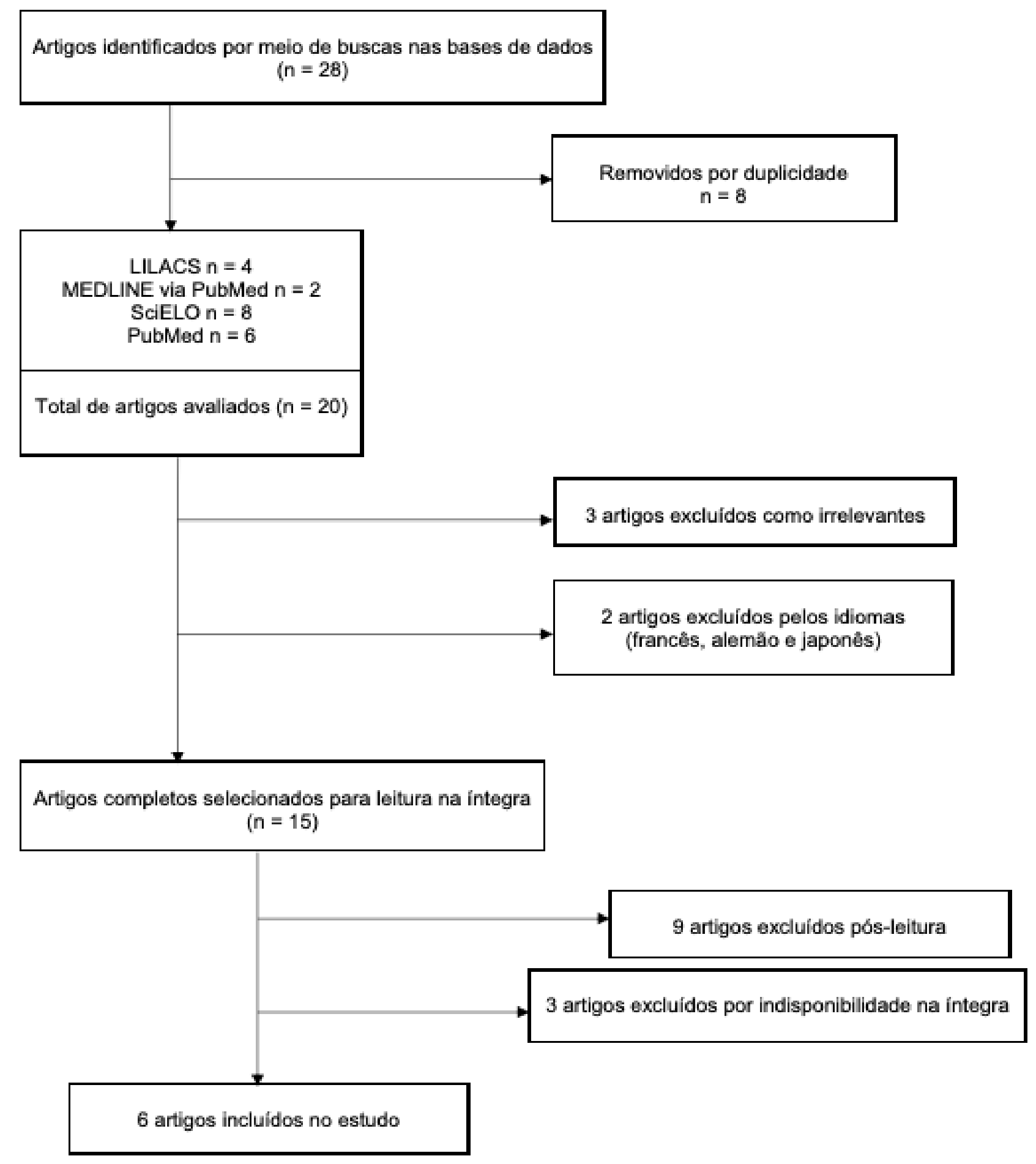

Fonte: Simão MCSA, et al., 2020.

\section{RESULTADOS}

A fim de avaliar a relação da PP como indicador de mortalidade na IC, bem como sua associação com a FEVE, foi somado o material obtido, 15 artigos, e feito uma leitura minuciosa, avaliando estudos que respondiam ao objetivo da revisão, no propósito de tabular e analisar criticamente os dados. Para isso, foram elaborados instrumentos de coleta de dados incluindo: título, ano de publicação, autor, objetivo e principais resultados (Quadro 1). A partir dos critérios de inclusão, 6 estudos foram incluídos, os quais estão referenciados no presente artigo. 
Quadro 1 - Informações principais dos estudos incluídos no artigo.

\begin{tabular}{|c|c|c|c|c|}
\hline Título & $\begin{array}{c}\text { Ano de } \\
\text { Publicação }\end{array}$ & Autor & Objetivo & Resultados \\
\hline $\begin{array}{l}\text { Different relationships between pulse } \\
\text { pressure and mortality in heart failure with } \\
\text { reduced, mid-range and preserved ejection } \\
\text { fraction }\end{array}$ & 2018 & Teng THK, et al. & $\begin{array}{l}\text { Avaliar a relação da PP com o } \\
\text { prognóstico da IC a partir da } \\
\text { FEVE. }\end{array}$ & $\begin{array}{l}\text { A relação entre PP e mortalidade na IC é influenciada pela FEVE. } \\
\text { No estudo, pacientes com FEVE reduzida tiveram maior taxa de } \\
\text { mortalidade em quintis menores de PP, enquanto a FEVE média } \\
\text { obteve maior índice tanto em quintis menores quanto maiores. Já } \\
\text { pacientes com FEVE preservada, tiveram maior mortalidade em } \\
\text { quintis menores e apenas uma tendência em quintis maiores. } \\
\text { Ademais, a PP baixa era mais presentes na FEVE reduzida e a } \\
\text { PP alta era mais presente na FEVE preservada. }\end{array}$ \\
\hline $\begin{array}{c}\text { Low pulse pressure is independently related } \\
\text { to elevated natriuretic peptides and } \\
\text { increased mortality in advanced chronic } \\
\text { heart failure }\end{array}$ & 2005 & Yildiran T, et al. & $\begin{array}{l}\text { Examinar o valor preditivo da PP } \\
\text { na mortalidade em pacientes com } \\
\text { ICC estável, mas avançada } \\
\text { (NYHA Classe III ou IV). }\end{array}$ & $\begin{array}{c}\text { A PP reduzida prediz aumento da mortalidade. A população } \\
\text { estudada foi dividida em quartis de PP, assim, em quartis mais } \\
\text { altos, em que a PP era maior que } 45 \mathrm{~mm} \text { Hg, a PA média foi um } \\
\text { melhor preditor. Entretanto, nos quartis abaixo, a PP foi um } \\
\text { marcador eficiente de mortalidade. }\end{array}$ \\
\hline $\begin{array}{l}\text { Differing prognostic value of pulse pressure } \\
\text { in patients with heart failure with reduced or } \\
\text { preserved ejection fraction: results } \\
\text { From the MAGGIC individual patient meta- } \\
\text { analysis }\end{array}$ & 2015 & Jackson CE, et al. & $\begin{array}{l}\text { Analisar o valor prognóstico da } \\
\text { pressão de pulso em pacientes } \\
\text { com ICFE preservada (FE } \geq 50 \% \text { ) } \\
\text { e ICFE reduzida (FE <30\%). }\end{array}$ & $\begin{array}{l}\text { No artigo, houve maior proporção de pacientes com ICFE reduzida } \\
\text { que obtinham } \mathrm{PP}<45 \mathrm{mmHg} \text {, quando comparado aos pacientes } \\
\text { com ICFE preservada, os quais apresentaram maior proporção de } \\
\text { casos com } \mathrm{PP}>65 \mathrm{mmHg} \text {. Além disso, a mortalidade foi maior em } \\
\text { pacientes com ICFE reduzida. Assim, a PP foi preditora } \\
\text { independente de mortalidade em pacientes com ICFE reduzida. }\end{array}$ \\
\hline $\begin{array}{l}\text { Association of arterial pulse pressure with } \\
\text { long-term clinical out comes in patients with } \\
\text { heart failure }\end{array}$ & 2016 & Laskey WK, et al. & $\begin{array}{l}\text { Avaliar a associação entre PP no } \\
\text { momento da alta hospitalar e } \\
\text { resultados adversos de pacientes } \\
\text { com espectro variado de IC. }\end{array}$ & $\begin{array}{l}\text { A PP avaliada no momento da alta hospitalar está associada a } \\
\text { resultados adversos ao longo de } 1 \text { ano em pacientes com IC. De } \\
\text { acordo com a PP, pacientes com ICFE reduzida, os quais tiveram } \\
\text { PP }<50 \mathrm{mmHg} \text {, apresentaram menor risco de mortalidade do que } \\
\text { pacientes com PP }>\text { ou }=50 \mathrm{mmHg} \text {. Já em pacientes com ICFE } \\
\text { preservada, } \mathrm{PP}<50 \mathrm{mmHg} \text { não apresentou associação com a } \\
\text { mortalidade, enquanto PP>0u=50mmHg apresentou relação entre } \\
\text { o aumento da PP e mortalidade, e entre PP e PAS. }\end{array}$ \\
\hline Low Pulse Pressure as a Predictor of Death & 2010 & Yildiran T, et al. & $\begin{array}{l}\text { Investigar o valor prognóstico da } \\
\text { pressão de pulso em pacientes } \\
\text { com insuficiência cardíaca. }\end{array}$ & $\begin{array}{l}\text { A baixa PP predita independentemente morte por causas } \\
\text { cardiovasculares em pacientes com IC moderada a reduzida. Os } \\
\text { pacientes foram divididos em quartis de PP e de NYHA. Nesse } \\
\text { estudo, quartis mais altos de PP relacionaram-se a um menor } \\
\text { nível de NYHA, assim, a PP alta foi preditora de morte em NYHA I } \\
\text { e II, enquanto a PP baixa foi preditora em NYHA III e IV. Ademais, } \\
\text { quartis mais altos de PP foram correlacionados a maior FEVE. }\end{array}$ \\
\hline $\begin{array}{l}\text { A low pulse pressure predicts mortality in } \\
\text { subjects with heart failure after na acute } \\
\text { myocardial infarction: a post-hoc analysis of } \\
\text { the CAPRICORN study }\end{array}$ & 2011 & Petrie CJ, et al. & $\begin{array}{l}\text { Examinar a importância da PP em } \\
\text { pacientes com sinais de disfunção } \\
\text { ventricular esquerda e infarto do } \\
\text { miocárdio recente, com ou sem } \\
\text { sintomas de IC. }\end{array}$ & $\begin{array}{l}\text { A PP baixa previu independentemente morte súbita e } \\
\text { cardiovascular em pacientes Killip II-IV, já em Killip I, não foi } \\
\text { preditor suficiente. }\end{array}$ \\
\hline
\end{tabular}

Fonte: Simão MCSA, et al., 2020. sintomas de IC. 
O estudo de Petrie CJ, et al. (2011), com amostra de 1955 pacientes pós-infarto do miocárdio, com idade média de 63 anos ( \pm 12 ) e FEVE menor ou igual a $40 \%$, com ou sem sinais de IC, buscou investigar a importância da PP em pacientes com sinais de disfunção ventricular esquerda e IAM recente, com ou sem sintomas de IC.

Os pacientes com alta PP foram classificados majoritariamente em Killip II-IV e associados a histórico de IC. Além disso, PP não era indicador suficiente de mortalidade por causas cardiovasculares em Killip I (PP média de 47mmHG), já em Killip II-IV (PP média de 49mmHg), a PP baixa previu independentemente morte cardiovascular e súbita. A taxa de mortalidade em Killip I foi 10,9\% e em Killip II-IV foi 19,4\%, por morte súbita ou cardiovascular. Pode-se concluir que indivíduos das classes Killip II-IV tiveram baixa PP como preditor independente de mortalidade por IC.

Em um estudo realizado por Voors AA, et al. (2005), objetivou o exame do valor preditivo da PP na mortalidade em pacientes com ICC estável, mas avançada. O estudo teve uma amostra de 1901 pacientes que foram divididos em grupos de acordo com a PP. Ademais, subdividiram a pressão de pulso em quartis ( [Q] quartil 1: $\mathrm{PP}<35 \mathrm{mmHg}$, quartil 2: PP 35 a $45 \mathrm{mmHg}$, quartil 3: PP 45 a $55 \mathrm{mmHg}$ e quartil 4: $\mathrm{PP}>55$ $\mathrm{mmHg}$ ) e apresentaram a taxa de óbito por quartil: Q1: 62.9\%; Q2: 65.1\%; Q3: 65.5\%; e Q4: 48,1\%. O estudo concluiu que a PP é um indicador prognóstico de fácil acesso clínico na ICC avançada, e a PP reduzida prediz independentemente $\mathrm{o}$ aumento da mortalidade.

O estudo de Jackson CE, et al. (2015) analisou o valor prognóstico da PP em pacientes com IC-FE (IC com fração de ejeção) preservada ( $\mathrm{FE} \geq 50 \%$ ) e ICFE reduzida ( $\mathrm{FE}<30 \%$ ). A amostra compreendia 27046 pessoas, com média de idade 65 anos. Nessa configuração, a proporção de pacientes com $\mathrm{PP}<45 \mathrm{mmHg}$ diferiu consideravelmente entre os dois grupos, visto que entre as pessoas com ICFE reduzida 39,9\% apresentaram $\mathrm{PP}<45 \mathrm{mmHg}$, enquanto, na população com IC-FE preservada, 20,5\% apresentaram $\mathrm{PP}<45$ $\mathrm{mmHg}$.

Ademais, a porcentagem de óbitos dentre a população com IC-FE reduzida foi maior em comparação com os pacientes de ICFE preservada. Com isso, conclui-se que em pacientes com IC-FE reduzida (particularmente naqueles com $\mathrm{FEVE}<30 \%$ ), nos quais a PP é mais baixa (especialmente $<53 \mathrm{mmHg}$ ), a PP prediz independentemente a mortalidade, principalmente naqueles com PAS reduzida $(<140 \mathrm{mmHg})$.

Outro artigo de Laskey WK, et al. (2016), analisou pacientes com IC previamente internados. Em uma população de 40421 pacientes, com média de 80 anos, diferentes resultados foram observados de acordo com a PP. Assim, nos pacientes com IC-FE reduzida, evidenciou-se que para uma $\mathrm{PP}<50 \mathrm{mmHg}$, a PP foi associada a resultados mais favoráveis, com $5,4 \%$ de redução do risco de mortalidade. Já para $P P \geq 50 \mathrm{mmHg}$, a PP aumentada foi associada com um maior risco de mortalidade, com 9,1\%.

Esses dados permitiram o estudo concluir que a PP avaliada no momento da alta hospitalar está associada a resultados adversos ao longo de 1 ano em pacientes com IC. Para pacientes com IC-FE reduzida e PP $<50$ $\mathrm{mmHg}$, o risco para desfechos adversos, como a mortalidade, aumenta à medida que a PP diminui. Por outro lado, para pacientes com PP>50 mmHg, o risco é potencializado com o aumento da PP.

Yildiran T, et al. (2010) investigou o valor prognóstico da PP em 225 pacientes com IC nas classificações funcionais NYHA I-IV. Os pacientes foram divididos em quartis de acordo com a pressão de pulso ( $\mathrm{PP}<35$, 35-45, 46-55, ou >55 mmHg) e com a classificação funcional NYHA (I, II, III, IV). Quanto aos quartis correlacionados ao NYHA, foram apresentados os seguintes resultados em relação a PP em mmHg: NYHA I (PP $45 \pm 10)$ - NYHA II (PP $46 \pm 15)$ - NYHA III (37 \pm 11$)$ - NYHA IV (31 \pm 9$)$. Já nos quartis relacionados a PP, foram evidenciados os seguintes resultados em relação a NYHA: PP < 35 (NYHA $3 \pm 0.8)$ - PP 35-45 (2.4 \pm 0.9) - PP 46-55 (2.3 \pm 0.7$)$ - PP>55 (2.1 \pm 0.7$)$. Além do mais, nos quartis referentes a PP, encontrou-se, em relação a FEVE, os resultados: $\mathrm{PP}<35(28 \% \pm 6 \%)$ - PP $35-45(32 \% \pm 6 \%)$ - PP $46-55(33 \% \pm 5 \%)-\mathrm{PP}>55$ $(36 \% \pm 5 \%)$.

O estudo mostrou que a baixa PP predita, independentemente, a morte por causas cardiovasculares em pacientes com IC moderada a avançada. A PP alta correlaciona-se a maior taxa de mortalidade em pacientes 
com classe NYHA I-II, enquanto a PP baixa, à maior taxa de mortalidade em pacientes com classe NYHA IIIIV. Neste artigo, os resultados revelaram que a PP e a FEVE são preditores independentes da morte por causa cardiovascular.

No artigo de Teng THK, et al. (2018), 36770 pacientes com insuficiência cardíaca foram avaliados durante 1 ano, a média de idade foi 74 ( \pm 12 anos). Os resultados evidenciaram uma associação entre mortalidade e PP, que foi alterada de acordo com a FEVE. Para avaliar a relação entre PP e FE foi subdividido os valores de PP em quintis: (quintil 1: 5 a $39 \mathrm{mmHg}$, quintil 2: 40 a $49 \mathrm{mmHg}$, quintil 3: 50 a $58 \mathrm{mmHg}$, quintil 4: 59 a 69 $\mathrm{mmHg}$ e quintil 5: 70 a $160 \mathrm{mmHg}$ ). Na fração de ejeção reduzida (30 a 39\%), a mortalidade foi maior em quintis menores.

Enquanto, na FE preservada ( $>50 \%$ ), observou-se o aumento dessa taxa nos quintis menores e uma tendência de aumento em quintis maiores. Ademais, foi constatado que a população com FE preservada era maior no quinto quintil. Por fim, a população com FE reduzida era maior nos primeiros quintis. Conclui-se que a associação entre PP e mortalidade na IC é influenciada pela FEVE, independentemente da PAS. Nesse cenário, a baixa PP foi independentemente correlacionada com a mortalidade na FE reduzida e na FE preservada. Já na PP alta foi vista uma tendência de crescimento da mortalidade em pacientes com FE média e preservada.

\section{DISCUSSÃO}

Na insuficiência cardíaca, a demanda metabólica não é atendida, ou é apenas mantida através de aumentos da PAD final. Nesse quadro, há uma diminuição do DC devido a disfunção do VE na fase diastólica, ou na fase sistólica, isto é, de contração ventricular, na qual o sangue será ejetado. Assim, a função em sístole pode ser estimada pela FEVE (MESQUITA ET, et al., 2004; SBC, 2018).

A IC sistólica, que representa cerca de $50 \%-60 \%$ dos casos, é caracterizada por uma perda de massa contrátil e aumento da rigidez do miocárdio por causa isquêmica e, na maioria dos casos, por dilatação do ventrículo devido a hipertrofia celular. Com isso, há uma FEVE reduzida, levando a redução do DC, do volume sistólico e ao aumento das pressões de enchimento (MONTERA MW, et al., 2009; SBC, 2018; MESQUITA $\mathrm{ET}$, et al., 2004).

Já na IC diastólica, que acomete $40 \%-50 \%$ dos pacientes, a contração do miocárdio é inalterada, o que além de não modificar a área do coração, permite uma FE preservada. Entretanto, por uma disfunção durante o relaxamento ou por uma redução da complacência ventricular, há restrição do volume da cavidade, resultando no aumento das pressões de enchimento. Ademais, enquanto a etiologia da IC com FEVE reduzida é, sobretudo, de origem isquêmica, a IC com FEVE preservada advém, principalmente, da hipertensão arterial (MONTERA, et al., 2009; MESQUITA ET, et al., 2004; SBC, 2018).

Além da fração de ejeção, outra medida relacionada à IC é a PP, que corresponde à diferença entre a PAS e a PAD, como também, é determinada pelo volume sistólico e complacência vascular, o que a torna capaz de predizer o risco de mortalidade por doenças cardiovasculares. A PP alta, na maioria dos casos, é reflexo do aumento da rigidez arterial que contribui para sobrecarga do ventrículo esquerdo (VE) e, por consequência, o aumento da pós carga do VE. A diminuição da complacência vascular é característica comum do envelhecimento, secundária a uma doença aterosclerótica generalizada, e no desenvolvimento de doenças crônicas como diabetes mellitus, hipertensão e dislipidemia (JACKSON CE, et al., 2015; VOORS AA, et al., 2005). Por outro lado, a diminuição da PP é consequência da baixa ejeção de sangue dos ventrículos e redução da função sistólica do VE resultando na perda de função cardíaca. Esse quadro é característico da IC, síndrome provocada pela disfunção ventricular, que possui sinais clássicos como baixa de fluxo sanguíneo, congestão pulmonar e remodelamento do VE (JACKSON CE, et al., 2015; VOORS AA, et al., 2005).

$\mathrm{Na}$ IC, a relação entre mortalidade e PP é alterada pela FEVE. Aqueles pacientes com FE preservada possuem função sistólica do VE inalteradas, entretanto existe uma disfunção do relaxamento ou anormalidade 
do enchimento ventricular e redução do volume sistólico. A IC-FE preservada parece ter relação com a maior $\mathrm{PP}$, visto que, muitas vezes, é acompanhada de outras condições como obesidade, diabetes mellitus, miocardiopatia hipertrófica e hipertensão que estão comumente relacionadas ao aumento da PP.

Já em pacientes com FE reduzida observamos perda da massa de miocárdio contrátil, redução do volume sistólico, baixo DC, aumento da PAD e hipotensão arterial. Dessa forma, o prognóstico é pior naqueles pacientes com PP reduzida (JACKSON CE, et al., 2015; MONTERA MW, et al., 2009). Outrossim, no estudo de Yildiran T, et al. (2010) existe uma concordância com o descrito, visto que, em seus resultados, pacientes com valores de PP baixa, eram classificados em NYHA III ou IV (NYHA I: PP $45 \pm 10$, NYHA II: PP $46 \pm 15$, NYHA III: PP $37 \pm 11$, NYHA IV: PP $31 \pm 9$ ), ou seja, possuíam estágios mais graves da IC e apresentavam sintomas característicos como: dispneia a grandes esforços e severas limitações.

Além disso, demonstra que indivíduos com PP baixa tendenciavam a ter uma FE menor (PP<35: FE $28 \%$ $\pm 6 \%$, PP $35-45$ FE 32\% $\pm 6 \%$, PP $46-55$ FE 33\% $\pm 5 \%$ e PP>55 FE 36\% $\pm 5 \%$ ), em concordância com o artigo de Voors et al., 2005 ( PP<45 mmHg: FE média 25\%, PP>45 mmHg: FE média 27\%) e Jackson CE, et al (2015) (IC-FE reduzida: PP média $52 \mathrm{mmHg}$, IC-FE preservada: PP média $62 \mathrm{mmHg}$ ). Portanto, concluise que existe uma associação positiva e independente entre a PP e FE.

Conforme Teng THK, et al. (2018), a FE influencia a relação entre PP e mortalidade pela IC, independentemente da PAS. Além disso, os resultados desse estudo, demonstraram que a taxa de mortalidade na IC-FE reduzida é maior entre pacientes com PP baixa (mortalidade quintil 1: 20,9\%, mortalidade no quintil 5: 15,3\%). Por outro lado, dentre indivíduos com IC-FE preservada, o número de óbitos foi maior naqueles com PP mais alta (mortalidade no quintil 1: $24,8 \%$, mortalidade no quintil $5: 18,4 \%$ ).

Nessa perspectiva, os estudos de Voors AA, et al. (2005) e de Jackson CE, et al. (2015), apresentam resultados semelhantes. Conforme Jackson CE, et al. (2015) a porcentagem de óbitos foi maior no grupo de pacientes com IC-FE reduzida (IC-FE reduzida: 23\%, IC-FE preservada: 17\%). Ademais, Voors AA, et al. (2005), relata que a taxa de sobrevida da população estudada foi maior em quartis com maior PP (Q1: 62.9\%; Q2: 65.1\%; Q3: 65.5\%; e Q4: 48,1\%.), embora em pacientes com PP maior que $45 \mathrm{mmHg}$, a PA apresentou maior acurácia para predizer o risco de óbito.

Já o estudo de Laskey WK, et al. (2016) chega a resultados diferentes, demonstrando que na IC-FE reduzida os indivíduos com PP menor que $45 \mathrm{mmHg}$ apresentaram melhor prognóstico (redução do risco de mortalidade em $5,4 \%$ ), já em pacientes com PP maior que $45 \mathrm{mmHg}$, o risco de eventos adversos foi maior (aumento do risco de mortalidade em 9,1\%). Além disso, na IC-FE preservada, para PP menor que $45 \mathrm{mmHg}$, não houve associação significativa com a mortalidade. Entretanto, reduções drásticas da PP impactou significativamente a mortalidade. Por outro lado, para PP maior que $45 \mathrm{mmHg}$ foi visto uma relação com um maior número de óbitos.

Por fim, Petrie CJ, et al. (2011), apresenta um estudo diferente dos demais ao comparar a PP com a classificação de KILLIP, em que são denotadas quatro classificações de acordo com gravidade da IC em pacientes com IAM. Dessa forma, Killip I indica disfunção sistólica do VE, mas sem sinais clínicos, II aponta indivíduos com estertores ou estalidos nos pulmões. Já a classificação III é visto como edema pulmonar franco e crepitação, e a IV é definida como choque cardiogênico ou hipotensão, além da evidência de hipoperfusão periférica. Dessa maneira, baixos valores de PP esteve presente na classificação de pacientes com Killip IIIV, sendo preditores independentes de mortalidade por IC.

Uma limitação deste estudo foi o baixo número de artigos selecionados para a realização da revisão integrativa, além disso, não foram encontrados ensaios clínicos randomizados controlados que utilizassem a pressão de pulso como fator preditor de mortalidade na IC. Diante disso, são necessários ainda muitos estudos randomizados para verificar a influência da pressão de pulso na mortalidade por IC.

\section{CONSIDERAÇÕES FINAIS}

Conclui-se que em pacientes com PP alta ou baixa, a taxa de mortalidade é maior, ou seja, a mortalidade geral aumenta em valores extremos da PP. A fim de analisar o uso da PP na IC foram selecionadas as 
seguintes variáveis: fração de ejeção, NYHA e Killip. Com relação a FEVE, em pacientes com IC-FE reduzida, a taxa de mortalidade foi maior naqueles com PP mais baixas, por outro lado, em indivíduos com IC-FE normal, a mortalidade aumenta à medida que a PP se eleva. Além disso, em relação ao NYHA, a PP alta foi associada com maior taxa de mortalidade em pacientes com classe NYHA I-II, já em pacientes com NYHA III-IV, a PP baixa foi associada a maior taxa de óbito. Ademais, em relação ao Killip, pacientes Killip II-IV tiveram a baixa PP como preditor independente de mortalidade por IC. Portanto, é importante salientar que a PP é um parâmetro de fácil obtenção, sem custos adicionais, que pode ser utilizado como marcador prognóstico em diversas situações clínicas, embora sejam necessários mais estudos que relacionem a PP como preditor independente de mortalidade em pacientes com IC.

\section{REFERÊNCIAS}

1. NAKA KK. Brachial pulse pressure in heart failure: simple to measure but complex to interpret. 2019; 40 (26): 8-10.

2. ALVIM R, et al. Rigidez Arterial: Aspectos Fisiopatológicos e Genéticos. International Journal of Cardiovascular Sciences. 2017; 30(5): 433-441.

3. ANGELI F, et al. Heart failure, pulse pressure and heart rate. International Journal of Cardiovascular Sciences. 2018

4. FAGARD R, et al. Is the predictive power of a low-pulse pressure independente of peak oxygen up take in advanced chronic heart failure? Journal of Human Hypertension. 2008; 22: 57-59.

5. FERREIRA AR, et al. Pulse pressure can predict mortality in advanced heart failure. Revista Portuguesa de Cardiologia 2016; 35 (4): 225-228.

6. JACKSON CE, et al. Differing prognostic value of pulse pressure in patients with heart failure with reduced or preserved ejection fraction: results from the MAGGIC individual patient meta-analysis. European Heart Journal. 2015; 36: 11061114.

7. LASKEY WK, et al. Association of Arterial Pulse Pressure with Long-Term Clinical Outcomes in PatientsWith Heart Failure. The American College of Cardiology Foundation. 2016;4(1).

8. MESQUITA ET, et al. Insuficiência cardíaca com função sistólica preservada, Brasil. Arquivos Brasileiros de Cardiologia, 2004; 82(5): 494-500.

9. MONTERA MW, et al. II Diretriz Brasileira de Insuficiência Cardíaca, Brasil. Arquivos Brasileiros de Cardiologia, 2009; 93(3): 2-65.

10.PELAZZA BB. Comparação entre os Valores da Pressão de Pulso em Hipertensos Controlados e não controlados. Revista Brasileira de Cardiologia. 2013; 26(6): 472-480.

11.PETRIE CJ, et al. A low pulse pressure predicts mortality in subjects with heart failure na acute myocardial infarction: a post-hoc analysis of the CAPRICORN study. Clinical Research in Cardiology. 2011; 101: 29-35.

12.ROHDE LEP, et al. Diretriz brasileira de insuficiência cardíaca crônica e aguda, Brasil. Arquivos Brasileiros de Cardiologia, 2018; 111(3): 436-539.

13.RODRIGUES A, et al. Pressão de Pulso como Marcador Prognóstico na Síndrome Coronariana Aguda. International Journalof Cardiovascular Sciences. 2015; 28(5): 409-416.

14.SÁ CR, et al. Pressão de Pulso como Marcador Prognóstico na Síndrome Coronariana Aguda. International Journal of Cardiovascular Sciences 2015; 28(5): 409-416.

15.TENG THK, et al. Different relationships between pulse pressure and mortality in heart failure with reduced, mid-range and preserved ejection fraction. International Journal of Cardiology, 2018; 254: 203-209.

16. YILDIRAN T, et al. Low pulse pressure as a predictor of death in patients with mild to advanced heart failure. Texas Heart Institute journal. 2010; 37(3): 284-290.

17.VOORS AA, et al. Low pulse pressure is independently related to elevated natriuretic peptides and increased mortality in advanced chronic heart failure. European Heart journal. 2005; 26 (17) 1759-1764.

18. ALBUQUERQUE D, et al. I Registro Brasileiro de Insuficiência Cardíaca- Aspectos Clínicos, Qualidade Assistencial e Desfechos Hospitalares. Arquivo Brasileiro Cardiol. 2015; 104 (6): 433-442.

19.MASTROCOLA LE, et al. Atualização da Diretriz Brasileira de Cardiologia Nuclear- 2020. Arquivo Brasileiro Cardiol. 2020; 114 (2): 325-429.

20.DOBRE D, et al. Heart Rate, Pulse Pressure and Mortality in Patients with Myocardial Infarction Complicated by Heart Failure. International Journal of Cardiology. 2018; 271: 181-185

21.SOCIEDADE BRASILEIRA DE CARDIOLOGIA. CARDIÔMETRO - Morte por Doenças Cardiovasculares no Brasil. Sociedade Brasileira de Cardiologia, 28 abr. 2020. Disponível em: < http://www.cardiometro.com.br/anteriores.asp >. Acesso em: 28 abr. 2020.

22.WORLD HEART FEDERATION. Heart Failure Infographic. World Heart Federation, 03 out. 2019. Disponível em: < https://www.world-heart-federation.org/resources/heart-failure-fact-sheet/>. Acesso em: 30 abr. 2020.

23. ORGANIZAÇÃO PAN-AMERICANA DA SAÚDE. Organização Mundial da Saúde divulga novas estatísticas mundiais de saúde. OPAS. Brasil, 17 mai. 2018. Disponível em: < https://www.paho.org/bra/index.php?option=com_content\&view=article\&id=5676:organizacao-mundial-da-saudedivulga-novas-estatisticas-mundiais-de-saude\&ltemid=843 >. Acesso em: 30 abr. 2020. 